\title{
Metabolic Acidosis in Preterm Infants is Associated with a Longer Length of Stay in the Neonatal Intensive Care Unit
}

\author{
Marika Paul $^{1} \cdot$ Jamie Partridge ${ }^{1} \cdot$ Bridget Barrett-Reis $^{1}\left(\mathbb{D} \cdot\right.$ Kaashif A. Ahmad $^{2} \cdot$ Pattabhi Machiraju $^{3}$. \\ Hemalatha Jayapalan ${ }^{3} \cdot$ Richard J. Schanler ${ }^{4}$
}

Published online: 23 January 2020

(c) The Author(s) 2020

\begin{abstract}
Introduction Preterm births account for disproportionately high healthcare costs, in large part due to expenses related to length of stay in the hospital neonatal intensive care unit (NICU). It is common for preterm infants to receive human milk fortifier (HMF) while in the NICU. Liquid HMF is available in both acidified and non-acidified formulations. A recent randomized clinical trial found that acidified HMF is associated with an increased incidence of metabolic acidosis, which may contribute to increased costs and longer NICU length of stay.

Objective The present study is a secondary analysis of these data, seeking to determine whether additional factors contribute to metabolic acidosis, whether metabolic acidosis is associated with longer hospital length of stay, and whether these associations contribute to the burden of hospital costs.

Methods The study sample consisted of 152 infants who were hospitalized in US NICUs. Multiple logistic regression was used to model the NICU length of stay. Data from the 2012 Healthcare Cost and Utilization Project (HCUP) Kids' Inpatient Database (KID) were used to calculate the average cost (charge) per day in a NICU. Costs (charges) were adjusted to \$US, year 2018 values, using the health Consumer Price Index.

Result Results indicated that acidified HMF was a strong predictor of metabolic acidosis, more so than gestational age or birth weight. Furthermore, metabolic acidosis was associated with incremental NICU costs (charges) of $\$$ US 19,002 $(\$ U S 65,462)$ per infant and longer NICU LOS.

Conclusion Future studies should further investigate factors that contribute to NICU length of stay and associated costs of care.

Trial Registration ClinicalTrials.gov: NCT02307760.
\end{abstract}

\section{Introduction}

Preterm births account for approximately $10 \%$ of all births but disproportionately greater healthcare costs in the USA [1-4]. Predictors of extended neonatal intensive care unit (NICU) stay, such as intracranial haemorrhage, necrotizing

Bridget Barrett-Reis

bridget.barrettreis@abbott.com

1 Abbott Nutrition R\&D, Bldg ES1 East, 2900 Easton Square Place, Columbus, OH, USA

2 Pediatrix Medical Group, North Central Baptist Hospital, San Antonio, TX, USA

3 Indegene, Tardeo, Mumbai, India

4 Neonatal-Perinatal Medicine, Cohen Children's Medical Center, New Hyde Park, NY, USA enterocolitis, bronchopulmonary dysplasia, and late-onset sepsis, all directly contribute to excess healthcare costs that average more than $\$$ US10,000 per case in hospital $[5,6]$. Moreover, these comorbidities are associated with higher post-discharge costs because of delays in long-term neurodevelopment [7]. Healthcare providers are therefore incentivized to reduce neonatal morbidities to provide better longterm outcomes for preterm infants and reduce the economic burden associated with the NICU hospital stay. Reducing the number of days an infant is hospitalized in the NICU contributes to cost savings in appropriate scenarios that do not jeopardize health outcomes [8].

Studies in other countries such as Sweden, the UK, Canada, and Germany have also reported risk factors for length of stay (LOS) in the NICU [9-13]. Seaton et al. [14] evaluated these studies combined with a few US studies in a systematic review and concluded that, in a simple model, 


\section{Key Points for Decision Makers}

Among preterm infants hospitalized in the neonatal intensive care unit (NICU), those who received acidified human milk fortifier had odds of developing metabolic acidosis more than 15 times higher than those of infants fed non-acidified human milk fortifier.

A diagnosis of metabolic acidosis was associated with an 8.5-days longer NICU stay (9.3 days from adjusted model).

The 9.3 additional days in the NICU was estimated to increase hospital costs associated with the development of metabolic acidosis by \$US19,002 (\$US65,462 charges).

inherent factors such as birth weight, gestational age, and sex were useful early indicators of LOS. The investigators also pointed out that understanding the predictors of LOS was necessary, not just for cost saving reasons but also for resource planning and parent counselling.

Metabolic acidosis occurs when blood $\mathrm{pH}$ is reduced, either by increased acid production or by inadequate clearance of acid by the kidneys. Metabolic acidosis may arise in preterm infants because of immature kidneys and a greater susceptibility to conditions that can lead to acidosis, such as infection or respiratory distress. Previous research has shown that metabolic acidosis is associated with poor developmental and neurologic outcomes in very low birth weight infants $[15,16]$. Subedi et al. [7] showed that increased NICU LOS had a highly significant negative relationship with longterm neurodevelopment outcomes at 9 months $(p<0.001)$ and 24 months $(p<0.01)$. Metabolic acidosis is also a risk factor for neonatal necrotizing enterocolitis, a condition that may require surgical intervention or can lead to sepsis and sometimes death [16-19].

Schanler et al. [20] recently reported that the incidence of metabolic acidosis was significantly greater in preterm infants who received acidified liquid human milk fortifier (HMF) than in those who received non-acidified liquid HMF. Additionally, the presence of metabolic acidosis itself was associated with a significantly longer NICU stay [20]. For the current study, we performed a secondary analysis of these data to identify whether additional factors were predictive of metabolic acidosis among the preterm infants who were included in the trial, excluding eight infants for whom NICU LOS was missing $(n=152)$. The relationship between metabolic acidosis and NICU LOS was modelled to assess hospital costs associated with this diagnosis. To our knowledge, no other published study has estimated costs associated with a diagnosis of metabolic acidosis in the NICU.

\section{Methods}

\subsection{Participants and Nutritional Intervention}

In the clinical trial, preterm infants with a birth weight of 700-1500 g who were born at or before 32 weeks' gestation and expected to survive with minimal morbidity were eligible for inclusion. Full study inclusion criteria for this US study are listed in further detail elsewhere [20]. Infants were randomized to receive a non-acidified liquid HMF or an acidified liquid HMF. LOS was not a primary outcome in the clinical trial; infants without a documented LOS were excluded from the current analysis. Of the 160 infants who participated in the trial, LOS data were unavailable for five infants in the acidified liquid HMF cohort and three infants in the non-acidified liquid HMF cohort, reducing the sample for the current analysis to $152(n=73$ and 79 infants in the acidified and non-acidified liquid HMF cohorts, respectively). Weight, feeding tolerance, and adverse events were assessed daily, and length, head circumference, and blood biochemistries were evaluated at study days 1,15 , and 29. LOS (time to discharge from the NICU to home) was followed. Metabolic acidosis was determined by physician report or met an a priori definition of a base excess of $<-6 \mathrm{mmol} / \mathrm{L}[20]$.

\subsection{Statistical and Cost Analysis}

Relationships between demographic and clinical factors and LOS were assessed using Chi squared tests for categorical variables and Spearman's correlations for continuous variables. Unadjusted analyses between demographic and clinical variables and metabolic acidosis were conducted using Chi squared tests for categorical variables and two-sample $t$ tests or Wilcoxon tests for continuous variables, depending on normality of the distribution.

A natural log transformation was performed on LOS to correct for a positively skewed distribution. Multiple linear regression employing backwards selection was used to model log-transformed LOS with a probability level of 0.05 as the threshold to remain in the model. Multiple logistic regression was used to model the incidence of metabolic acidosis. Healthcare costs were calculated using data from the 2012 Healthcare Cost and Utilization Project (HCUP) Kids' Inpatient Database (KID), which were the most recent at the time the study was conducted [10]. HCUP KID includes charges for each infant's NICU stay. Because charges reflect the billed expenses for a NICU stay, charges were converted to costs using the HCUP cost to charge file and adjusted for 
inflation to \$US, year 2018 values, using the healthcare Consumer Price Index [21, 22]. Costs more accurately reflect the expenses hospitals incur and the amounts health insurance companies reimburse for services provided. International Classification of Diseases, Ninth Revision (ICD-9) diagnosis and procedural codes were used to identify infants in the 2012 HCUP KID database who were born at or before 32 weeks' gestation, weighed $<1500 \mathrm{~g}$ at birth, were admitted to a NICU, and received parenteral feeding. Infants who died before NICU discharge were excluded. All analyses were performed using SAS version 9.3 software (SAS Institute, Inc., Cary NC, 2011).

The initial regression model predicting log-transformed LOS included gestational age at birth, 5-minute Apgar score, sex, race, ethnicity, weight, age at study day 1 (defined as the first day HMF was received), diagnosis of metabolic acidosis, and treatment group. Statistically significant covariates were identified using backwards selection. In models with log-transformed dependent variables, the coefficients were interpreted to approximate the percentage change in the dependent variable per unit change in the independent variable, holding other predictors constant [23].

\section{Results}

The characteristics of the 152 infants included in this analysis are described in Table 1. Infants who developed metabolic acidosis were more likely to be born earlier in gestation ( 28.3 vs. 29.4 weeks, $p=0.002$ ) and were younger at study day 1 (chronological age in days) than infants without metabolic acidosis ( 8.6 vs. $10.8, p=0.02$ ). The distributions of gestational ages plotted in Fig. 1 illustrate that the incidence of metabolic acidosis was much higher in infants born very preterm ( $<28$ weeks) than in those born at later, though still preterm, gestational ages (31.3 vs. $10.8 \%, p=0.01$ ).

The incidence of metabolic acidosis was 5\% (4 of 79) in the non-acidified liquid HMF cohort versus 26\% (19 of 73) in the acidified liquid HMF cohort. Of infants born before 28 weeks, $47 \%$ ( 8 of 17) in the acidified liquid HMF cohort and 13\% ( 2 of 15) in the non-acidified liquid HMF cohort developed metabolic acidosis. For the non-acidified liquid HMF cohort, metabolic acidosis occurred in the two youngest infants included in the study (born at 24 weeks and 4 days and at 25 weeks and 3 days) (Fig. 1).

Logistic regression modelling with backwards selection revealed three statistically significant predictors of metabolic acidosis (Table 2). Younger gestational age (weeks at birth) and younger age at study day 1 (days after birth) were both risk factors. Acidification of HMF was strongly correlated with metabolic acidosis; the adjusted odds for infants in the acidified liquid HMF group was 15.6 times that of infants in the non-acidified liquid HMF group $(p=0.0002)$.

\subsection{Length of Hospital Stay}

In unadjusted univariate analyses (Table 3), LOS was negatively correlated with weight at birth, weight at study day 1 , and gestational age at birth. Presence of metabolic acidosis was also significantly correlated with hospital LOS: infants diagnosed with metabolic acidosis were hospitalized for an average of 8.5 days longer than infants without metabolic acidosis (65.5 vs. 57.0 days, $p=0.03$ ). LOS in the non-acidified liquid HMF group was lower than in the acidified liquid HMF group, but this was not statistically significant $(57.6 \pm 21.7$ vs. $58.9 \pm 19.8$ days, $p=0.71)$. Although gestational age was a strong predictor of LOS, it was confounded with other variables of interest, e.g. birth weight, intraventricular haemorrhage, and necrotizing enterocolitis; we therefore excluded it from our multivariable analysis. Greater weight at study day 1 was predictive of shorter NICU stays, but older age at study day 1 was predictive of longer NICU stays. Each additional day of age at study day 1 was estimated to increase LOS by $3 \%(p<0.0001)$. Black infants were predicted to have a $13 \%$ shorter LOS than White infants $(p=0.008)$.

\subsection{Economic Costs Associated with Metabolic Acidosis-Related Length of Stay}

Finally, differences in LOS associated with metabolic acidosis were translated into costs using data from the 2012 HCUP KID. The average cost (charge) per day, adjusted to \$US, year 2018 values, among infants who met inclusion criteria was \$US2036 (\$US7014 charge). Using the coefficients produced by the model in Table 3, a diagnosis of metabolic acidosis was therefore estimated to increase the cost (charge) of a NICU stay for an infant of average age and weight at study day 1 by $\$$ US19,002 (\$US65,462 charge).

\section{Discussion}

Among preterm infants who participated in a clinical trial to evaluate HMF liquid formulations, those who were fed acidified liquid HMF had 15 times the odds of developing metabolic acidosis compared with infants fed non-acidified liquid HMF. Susceptibility to metabolic acidosis was highest in the youngest infants. However, acidification of HMF appeared to be a stronger factor than prematurity alone: among babies born very preterm (before 28 weeks), the incidence of metabolic acidosis was $47 \%$ in the acidified liquid HMF cohort, more than three times higher than the incidence in the nonacidified liquid HMF group (13\%). Combined, these findings suggest that consumption of an acidified liquid HMF substantially increases the risk of developing metabolic acidosis. A diagnosis of metabolic acidosis can have far-reaching 
Table 1 Study population

\begin{tabular}{|c|c|c|c|}
\hline Variable & $\begin{array}{l}\text { Metabolic acidosis absent } \\
(n=129)\end{array}$ & $\begin{array}{l}\text { Metabolic acidosis present } \\
(n=23)\end{array}$ & $p$ value \\
\hline Treatment group & & & 0.0003 \\
\hline Acidified liquid HMF group & $54(74.0)$ & $19(26.0)$ & \\
\hline Non-acidified liquid HMF group & 75 (94.9) & $4(5.1)$ & \\
\hline Sex & & & 0.78 \\
\hline Males & $57(82.6)$ & $12(17.4)$ & \\
\hline Female & $72(86.8)$ & $11(13.3)$ & \\
\hline Race & & & 0.55 \\
\hline White & $88(87.1)$ & $13(12.9)$ & \\
\hline Black & $33(80.5)$ & $8(19.5)$ & \\
\hline Other & $8(80.0)$ & $2(20.0)$ & \\
\hline Ethnicity & & & 0.89 \\
\hline Hispanic & $24(85.7)$ & $4(14.3)$ & \\
\hline Non-Hispanic & $105(84.7)$ & $19(15.3)$ & \\
\hline Gestational age (weeks) & $29.4 \pm 1.6$ & $28.3 \pm 1.7$ & 0.002 \\
\hline Weight at birth (g) & $1209.9 \pm 190.0$ & $1154.6 \pm 237.4$ & 0.22 \\
\hline Apgar score $<7$ & & & 0.70 \\
\hline Yes & $12(80.0)$ & $3(20.0)$ & \\
\hline No & $117(85.4)$ & $20(14.6)$ & \\
\hline Age at study day 1 (days) & $10.8 \pm 5.1$ & $8.6 \pm 2.0$ & 0.02 \\
\hline Length of stay (days) & $57.0 \pm 20.7$ & $65.5 \pm 19.7$ & 0.03 \\
\hline Weight at study day 1 (g) & $1220.3 \pm 188.2$ & $1114.8 \pm 257.0$ & 0.11 \\
\hline Intraventricular haemorrhage & & & 0.55 \\
\hline Yes & $21(80.8)$ & $5(19.2)$ & \\
\hline No & $108(85.7)$ & $18(14.3)$ & \\
\hline Necrotizing enterocolitis & & & 0.28 \\
\hline Yes & $1(50.0)$ & $1(50.0)$ & \\
\hline No & $128(85.3)$ & $22(14.7)$ & \\
\hline Sepsis & & & 1.0 \\
\hline Yes & $4(100.0)$ & $0(0.0)$ & \\
\hline No & $125(84.5)$ & $23(15.4)$ & \\
\hline
\end{tabular}

Data are presented as mean \pm standard deviation or $\mathrm{N}(\%)$ unless otherwise indicated $H M F$ human milk fortifier

effects, as data show that metabolic acidosis is associated with poor developmental and neurologic outcomes in very low birth weight infants $(<1500 \mathrm{~g})[15,16]$. As previously mentioned, metabolic acidosis is also a risk factor for neonatal necrotizing enterocolitis [16-19].

We also examined factors that predicted LOS and found that older chronological age at study day 1 was predictive of longer NICU hospitalizations: each additional day was estimated to increase LOS by $3 \%$. This is consistent with results of the clinical trial analysis, which showed that older age at study day 1 was correlated with risk factors such as lower birth weight, lower gestational age, and more days to regain birth weight. In adjusted models, infants who developed metabolic acidosis had an average increase in LOS of 9.3 days compared with infants who did not develop metabolic acidosis. Longer NICU stays increase costs and may also affect infant development. Subedi et al. [7] showed that increasing NICU LOS was significantly associated with long-term neurodevelopment outcomes. This difference in LOS in the present analyses is estimated to translate to an increase of \$US19,002 (\$US65,462 charges) in hospitalization costs associated with developing metabolic acidosis.

US hospitals have a financial incentive to discharge infants from the NICU. Nearly half of all births are paid for by Medicaid, which reimburses healthcare providers and hospitals at lower rates than does private insurance [24]. Medicaid-enrolled mothers are more likely to deliver preterm infants than women who are privately insured [25]. Reducing NICU LOS may also be important to other stakeholders, including contracted NICU organizations that compete in the neonatology space and private health insurance companies that continually focus on cost containment. 


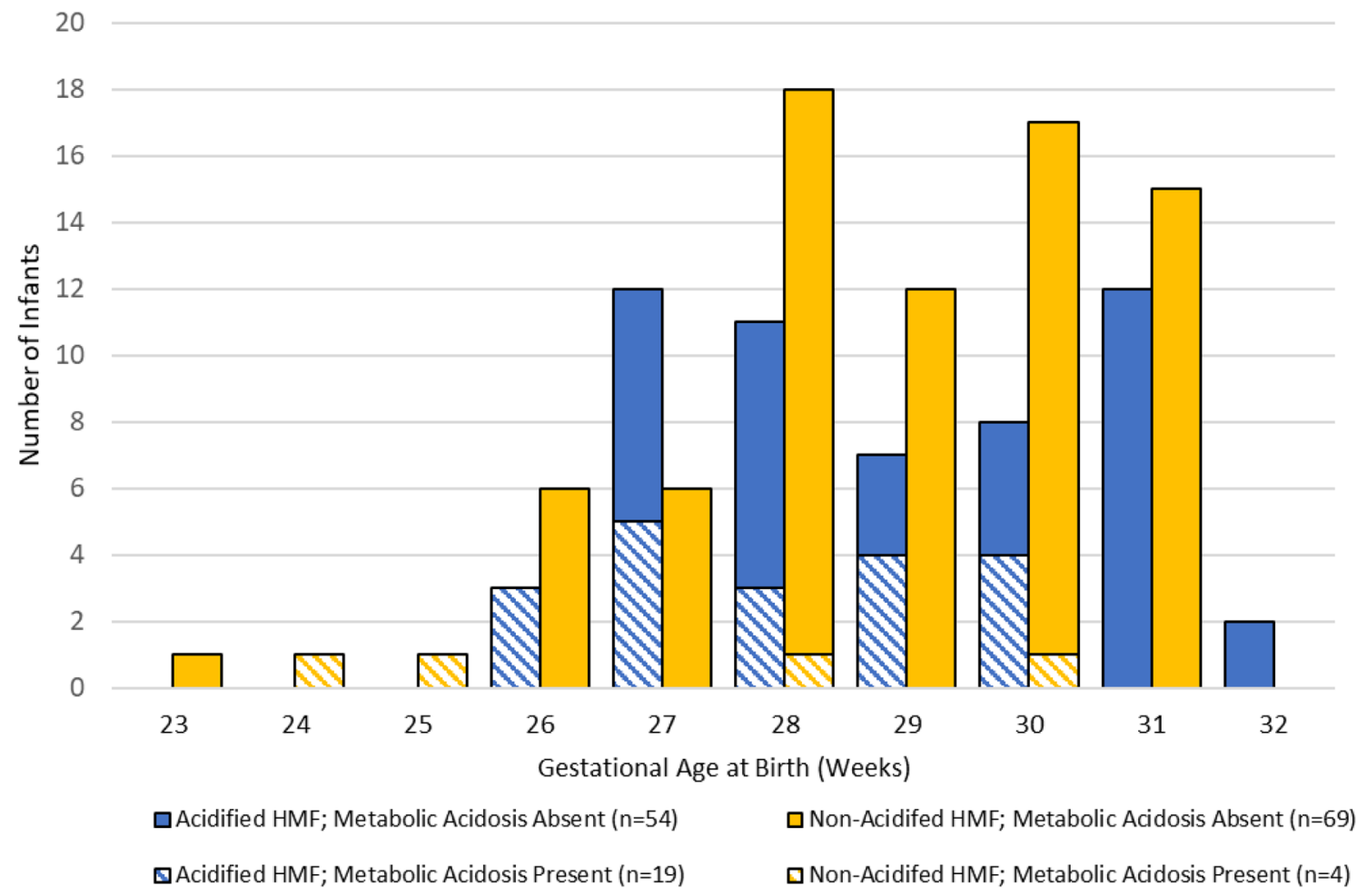

Fig. 1 Metabolic acidosis diagnoses by human milk fortifier (HMF) treatment group and gestational age

Table 2 Logistic model predicting metabolic acidosis

\begin{tabular}{|c|c|c|c|c|c|c|}
\hline \multirow[t]{2}{*}{ Variable } & \multicolumn{3}{|l|}{ Univariable models } & \multicolumn{3}{|l|}{ Multivariable model } \\
\hline & OR (95\% CI) & $p$ value & C-Stat & OR $(95 \% \mathrm{CI})$ & $p$ value & C-Stat ${ }^{\mathrm{a}}$ \\
\hline Weight on study day $1(\mathrm{~kg})$ & $0.08(0.009-0.72)$ & 0.024 & 0.605 & & & 0.90 \\
\hline Age on study day 1 (days) & $0.84(0.72-0.99)$ & 0.038 & 0.612 & $0.62(0.46-0.82)$ & 0.0008 & \\
\hline Acidified liquid HMF & $6.60(2.12-20.50)$ & 0.0011 & 0.704 & $15.58(3.68-66.06)$ & 0.0002 & \\
\hline Gestational age (weeks) & $0.67(0.51-0.88)$ & 0.003 & 0.695 & $0.38(0.25-0.60)$ & $<0.0001$ & \\
\hline Birthweight (kg) & $0.26(0.03-2.25)$ & 0.22 & 0.563 & & & \\
\hline Male & $1.38(0.57-3.35)$ & 0.48 & 0.540 & & & \\
\hline Race & & & 0.559 & & & \\
\hline Black & $1.64(0.62-4.32)$ & 0.32 & & & & \\
\hline Other & $1.69(0.32-8.86)$ & 0.53 & & & & \\
\hline Hispanic & $0.92(0.29-2.96)$ & 0.89 & 0.506 & & & \\
\hline Apgar score $<7$ & $1.46(0.38-5.65)$ & 0.58 & 0.519 & & & \\
\hline Intraventricular haemorrhage & $1.43(0.48-4.27)$ & 0.52 & 0.527 & & & \\
\hline
\end{tabular}

$C I$ confidence interval, $H M F$ human milk fortifier, $O R$ odds ratio

${ }^{a}$ This C-statistic represents the multivariable model
Similar economic forces would likely come into play for infants in the NICU outside of the USA.

While the results of this study are compelling, we note the following limitations. Though HCUP is a nationally representative and reliable data source, the costs are imputed. Ideally, these analyses would be conducted with cost data from participating institutions. NICU charges are generally per diem, so, even when using a cost-to-charge ratio, it is not possible to determine the actual cost of the resources used to care for premature infants. Future studies should employ larger sample sizes to allow for more extensive analyses of factors that contribute to NICU LOS and associated costs of care. 
Table 3 Multivariable regression model predicting log-transformed length of stay

\begin{tabular}{|c|c|c|c|c|c|c|}
\hline \multirow[t]{2}{*}{ Variable } & \multicolumn{3}{|c|}{ Univariable model } & \multicolumn{3}{|c|}{ Multivariable model } \\
\hline & Coefficient (SE) & $p$ value & $R$-squared & Coefficient (SE) & $p$ value & $R$-squared ${ }^{\mathrm{a}}$ \\
\hline Weight on study day $1(\mathrm{~kg})$ & $-0.80(0.13)$ & $<0.0001$ & 0.2121 & $-0.87(0.11)$ & $<0.0001$ & 0.4464 \\
\hline Age on study day 1 (days) & $0.03(0.006)$ & $<0.0001$ & 0.1405 & $0.03(0.01)$ & $<0.0001$ & \\
\hline Acidified liquid HMF & $0.03(0.06)$ & 0.54 & 0.0025 & & & \\
\hline Metabolic acidosis & $0.16(0.08)$ & 0.047 & 0.0261 & $0.15(0.06)$ & 0.01 & \\
\hline Birthweight (kg) & $-0.98(0.12)$ & $<0.0001$ & 0.3068 & & & \\
\hline Male & $0.10(0.06)$ & 0.09 & 0.0194 & & & \\
\hline \multicolumn{7}{|l|}{ Race } \\
\hline Black & $-0.11(0.06)$ & 0.08 & 0.0221 & $-0.13(0.05)$ & 0.008 & \\
\hline Other & $0.03(0.12)$ & 0.80 & & $-0.12(0.09)$ & 0.18 & \\
\hline Hispanic & $-0.05(0.07)$ & 0.53 & 0.0026 & & & \\
\hline Apgar score $<7$ & $0.17(0.09)$ & 0.08 & 0.0208 & & & \\
\hline Sepsis & $0.30(0.18)$ & 0.09 & 0.0187 & & & \\
\hline Intraventricular haemorrhage & $0.07(0.08)$ & 0.39 & 0.0050 & & & \\
\hline
\end{tabular}

$H M F$ human milk fortifier, $S E$ standard error

${ }^{a}$ This $R$-squared represents the multivariable model

Author contributions MP, JP, BBR, KA, PM, HJ, RS. MP, JP, and BBR conceived the study and were in charge of the overall direction and planning. MP, JP, BBR, PM, and HJ designed the model and computational framework. MP, PM, and HJ analysed the data. All study authors provided input into the interpretation of the results and contributed to the writing of the manuscript.

Data Availability Statement The 2012 HCUP KID dataset is neither a free nor publicly available dataset. It was approved for use for this study under a data use agreement (DUA) that does not allow the data to be shared with anyone not included on the DUA. All relevant data are within the manuscript and its supporting information files.

\section{Compliance with Ethical Standards}

Conflict of interest Dr. Partridge, Dr. Barrett-Reis and Dr. Paul receive salary support from Abbott Nutrition. Dr. Machiraju and Dr. Jayapalan received compensation as statistical consultants from Abbott Nutrition. Dr. Schanler and Dr. Ahmad received research funding from Abbott Nutrition for the clinical trial. Data collected during the clinical trial were used for the current study.

Open Access This article is licensed under a Creative Commons Attribution-NonCommercial 4.0 International License, which permits any non-commercial use, sharing, adaptation, distribution and reproduction in any medium or format, as long as you give appropriate credit to the original author(s) and the source, provide a link to the Creative Commons licence, and indicate if changes were made. The images or other third party material in this article are included in the article's Creative Commons licence, unless indicated otherwise in a credit line to the material. If material is not included in the article's Creative Commons licence and your intended use is not permitted by statutory regulation or exceeds the permitted use, you will need to obtain permission directly from the copyright holder.To view a copy of this licence, visit http://creativecommons.org/licenses/by-nc/4.0/.

\section{References}

1. Martin JA, Hamilton BE, Osterman MJK. Births in the United States, 2017. NCHS Data Brief. 2018;318:1-8.

2. Grosse SD, Waitzman NJ, Yang N, et al. Employer-sponsored plan expenditures for infants born preterm. Pediatrics. 2017;140(4):e20171078.

3. Services NPISQA. Special Care Nursery Admissions. 2011. https ://www.marchofdimes.org/peristats/pdfdocs/nicu_summary_final .pdf. Accessed 17 Sept 2018

4. Harrison W, Goodman D. Epidemiologic trends in neonatal intensive care, 2007-2012. JAMA Pediatr. 2015;169:855-62.

5. Johnson TJ, Patel AL, Jegier BJ, et al. Cost of morbidities in very low birth weight infants. J Pediatr. 2013;162(2):243-9.

6. Hintz SR, Bann CM, Ambalavanan N, et al. Predicting time to hospital discharge for extremely preterm infants. Pediatrics. 2010;125(1):e146-54.

7. Subedi D, DeBoer MD, Scharf RJ. Developmental trajectories in children with prolonged NICU stays. Arch Dis Child. 2017;102:29-34.

8. Silber JH, Lorch SA, Rosenbaum PR, et al. Time to send the preemie home? Additional maturity at discharge and subsequent health care costs and outcomes. Health Serv Res. 2009;44:444-63.

9. Altman M, Vanpée M, Cnattingius S, et al. Moderately preterm infants and determinants of length of hospital stay. Arch Dis Child Fetal Neonatal Ed. 2009;94:F414-8.

10. Manktelow B, Draper ES, Field C, et al. Estimates of length of neonatal stay for very premature babies in the UK. Arch Dis Child Fetal Neonatal Ed. 2010;95:F288-92.

11. Zernikow B, Holtmannspötter K, Michel E, et al. Predicting length-of-stay in preterm neonates. Eur J Pediatr. 1999;158:59-62.

12. Berry MA, Shah PS, Brouillette RT, et al. Predictors of mortality and length of stay for neonates admitted to children's hospital neonatal intensive care units. J Perinatol. 2008;28:297-302.

13. Hinchliffe SR, Seaton SE, Lambert PC, et al. Modelling time to death or discharge in neonatal care: an application of competing risks. Paediatr Perinat Epidemiol. 2013;27:426-33. 
14. Seaton SE, Barker L, Jenkins D, et al. What factors predict length of stay in a neonatal unit: a systematic review. BMJ. 2016;6:e010466.

15. Goldstein RF, Thompson RJ Jr, Oehler JM, Brazy JE. Influence of acidosis, hypoxemia, and hypotension on neurodevelopmental outcome in very low birth weight infants. Pediatrics. 1995;95:238-43.

16. Morgan JL, Nelson DB, Casey BM, et al. Impact of metabolic acidemia at birth on neonatal outcomes in infants born before 34 weeks' gestation. J Matern Fetal Neonatal Med. 2017;30(16):1902-5.

17. Henry MC, Moss RL. Neonatal necrotizing enterocolitis. Semin Pediatr Surg. 2008;17:98-109.

18. Fitzgibbons SC, Ching Y, Yu D, et al. Mortality of necrotizing enterocolitis expressed by birth weight categories. J Pediatr Surg. 2009;44:1072-5 (discussion 1075-6).

19. Sharma R, Hudak ML. A clinical perspective of necrotizing enterocolitis: past, present, and future. Clin Perinatol. 2013;40:27-51.
20. Schanler RJ, Groh-Wargo SL, Barrett-Reis B, et al. Improved outcomes in preterm infants fed a nonacidified liquid human milk fortifier: a prospective randomized clinical trial. J Pediatr. 2018;202:31-7.

21. Agency for Health Care Research and Quality. KID Database Documentation. Healthcare Cost and Utilization Project (HCUP). 2018. www.hcup-us.ahrq.gov/db/nation/kid/kiddbdocumentation. jsp. Accessed 5 Sept 2018

22. Statistics USDoLBoL. Databases, Tables and Calculators by Subject. 2018. https://www.bls.gov/data/inflation_calculator.htm. Accessed 1 Sept 2018

23. Wooldridge JM. Introductory econometrics: a modern approach. Toronto: Nelson Education; 2015.

24. NCHS Data Brief. https://www.cdc.gov/nchs/data/databriefs/ db318.pdf. Accessed 15 Jan 2019.

25. Markus AR, et al. Examining the association between medicaid coverage and preterm births using 2010-2013 National Vital Statistics Birth Data. J Child Poverty. 2017;23(1):79-94. 